\title{
Z-Score Functions of Dual Hesitant Fuzzy Set and Its Applications in Multi-Criteria Decision Making
}

\author{
Zahari Md Rodzi $^{1,2, *}$, Abd Ghafur Ahmad ${ }^{2}$, Nur Sa'aidah Ismail ${ }^{3}$, Wan Normila Mohamad ${ }^{4}$, Sarahiza \\ Mohmad $^{1}$ \\ ${ }^{1}$ Faculty of Computer and Mathematical Science, Universiti Teknologi MARA Campus Seremban, 70300 Seremban, Negeri Sembilan, \\ Malaysia \\ ${ }^{2}$ School of Mathematical Science, Faculty of Science and Technology, Universiti Kebangsaan Malaysia, 43600 UKM, Bangi, Selangor, \\ Malaysia \\ ${ }^{3}$ Faculty of Computer and Mathematical Science, Universiti Teknologi MARA Campus Dengkil, 43800 Dengkil, Selangor, Malaysia \\ ${ }^{4}$ Faculty of Business and Management, Universiti Teknologi MARA Campus Seremban, 70300 Seremban, Negeri Sembilan, Malaysia
}

Received January 21, 2021; Revised March 17, 2021; Accepted April 18, 2021

\section{Cite This Paper in the following Citation Styles}

(a): [1] Zahari Md Rodzi, Abd Ghafur Ahmad, Nur Sa'aidah Ismail, Wan Normila Mohamad, Sarahiza Mohmad, "Z-Score Functions of Dual Hesitant Fuzzy Set and Its Applications in Multi-Criteria Decision Making," Mathematics and Statistics, Vol. 9, No. 3, pp. 225 - 232, 2021. DOI: 10.13189/ms.2021.090303.

(b): Zahari Md Rodzi, Abd Ghafur Ahmad, Nur Sa'aidah Ismail, Wan Normila Mohamad, Sarahiza Mohmad (2021). Z-Score Functions of Dual Hesitant Fuzzy Set and Its Applications in Multi-Criteria Decision Making. Mathematics and Statistics, 9(3), 225 - 232. DOI: 10.13189/ms.2021.090303.

Copyright $@ 2021$ by authors, all rights reserved. Authors agree that this article remains permanently open access under the terms of the Creative Commons Attribution License 4.0 International License

\begin{abstract}
Dual hesitant fuzzy set (DHFS) consists of two parts: membership hesitant function and non-membership hesitant function. This set supports more exemplary and flexible access to set degrees for each element in the domain and can address two types of hesitant in this situation. It can be considered a powerful tool for expressing uncertain information in the decision-making process. The function of z-score, namely z-arithmetic mean, z-geometric mean, and z-harmonic mean, has been proposed with five important bases, these bases are hesitant degree for dual hesitant fuzzy element (DHFE), DHFE deviation degree, parameter $\alpha$, (the importance of the hesitant degree), parameter $\beta$, (the importance of the deviation degree) and parameter $\vartheta$, (the importance of membership (positive view) or non-membership (negative view). A comparison of the $z$-score with the existing score function was made to show some of their drawbacks. Next, the z-score function is then applied to solve multi-criteria decision making (MCDM) problems. To illustrate the proposed method's effectiveness, an example of MCDM specifically in pattern recognition has been shown.
\end{abstract}

Keywords Z-Arithmetic Mean, Z-Geometric Mean, Z-Harmonic Mean, Score Function, DHFS, MCDM, DHFE

\section{Introduction}

Dual hesitant fuzzy set (DHFS)[1] is a recent generalization of fuzzy set (FS)[2] which consists of membership hesitant function and non-membership hesitant function' DHFS confronts several different potential values that indicate whether certainty or ambiguity is the epistemic degree. This includes FS, intuitionistic fuzzy set (IFS)[3], [4] and hesitant fuzzy set (HFS)[5], [6] so that ambiguous knowledge can be treated more flexibly in the decision-making process. Numerous researchers applied DHFS to solve problems in multi-criteria decision making (MCDM)[7]-[14]. Usually all forms of MCDM need to evaluate and identify the DHFE information from a single expert or community expert for better potential option. It is therefore very necessary to differentiate between any two DHFEs and rank them efficiently. One the technique for differentiate the DHFE is score functions. Chen [15] proposed a score function and accuracy function to rank DHFE based on average value of DHFE, while Ren et al. [16] suggested that the score feature relies on three aspects: (1) the hesitant degree of membership and non-membership functions; (2) 
the mean hesitant degree of DHFE arising from one minus mean membership and non-membership values; (3) the non-membership feature should be deemed more significant. Numerous researchers applied the score functions of HFS and DHFS in their research [17], [18], [27]-[29], [19]-[26]. While many excellent contributions to score functions of varying types were made in the sense of DHFS, certain critical issues remain to be tackled. Are all decision makers (DMs) prefer the negative view (non-membership) compared to positive view (membership)? How important is the hesitant degree in determining the score for DHFE? This research aims to examine the essence of DHFE in DHFS score functions from a DM perspective and analyze the versatility of the newly proposed score functions.

The rest of this paper is structured as follows. In the preliminaries section, we present several simple definitions of HFS, DHFS and score functions of DHFE. For the next section, we propose $\mathrm{z}$-score function namely z-arithmetic mean, z-geometric mean and z-harmonic mean and make some sensitivity analysis and compare them with existing score functions. Then, we suggest weighted $\mathrm{z}$-score algorithms to solve MCDM problems, in particular pattern recognition in the DHFS environment. Lastly, we conclude the paper and provide some relevant remarks.

\section{Materials and Methods}

Definition 1. [5], [6] Given a fixed set $X$, then a HFS denoted as $K$ on $X$ is a term of a function that applied to $X$ which returns a subset of $[0,1]$. The HFS can be written as

$$
K=(\langle x, h(x)\rangle \mid x \in X)
$$

where $h(x)$ is a set of some value in $[0,1]$, on behalf of the probable membership degree of the element $x \in X$ for the set $K$. For simplicity, [30] called $h=h(x)$ a hesitant fuzzy element (HFE) and $H$ is the set of all HFEs.

Definition 2. [1] Given a fixed set $X$, then a DHFS $D$ on $X$ is a term of a function that when applied to $X$ returns a subset of $[0,1]$. The DHFS can be expressed in mathematical symbols as

$$
D=(\langle x, h(x), g(x)\rangle \mid x \in X)
$$

in which $h(x)$ and $g(x)$ are two sets of some values in $[0,1]$, denoting the possible membership degree and non-membership degree for the elements $x \in X$ to the set $D$, respectively with the conditions $0 \leq \gamma, \eta \leq 1$ and $0 \leq \gamma^{+}+\eta^{+} \leq 1 \quad$ where $\quad \gamma \in h(x) \quad$ and $\quad \eta \in g(x)$ $\gamma^{+} \in h^{+}(x)=\cup_{\gamma \in h(x)} \max \{y\}$ and $\eta^{+} \in g^{+}(x)=\cup_{\eta \in g(x)} \max \{\eta\}$.

For convenience, the pair $d=\{h(x), g(x)\}$ is called
DHFE denoted by $d=\{h, g\}$.

Definition 3. [1]. Let two DHFEs $d_{1}=\left\{h_{1}, g_{1}\right\}$ and $d_{2}=\left\{h_{2}, g_{2}\right\}$. The score function and accuracy function of $d_{i}$ are defined as $s_{i}=\frac{\sum \gamma}{\# h_{i}}-\frac{\sum \eta}{\# g_{i}}$, and $p_{i}=\frac{\sum \gamma}{\# h_{i}}+\frac{\sum \eta}{\# g_{i}}$ respectively. With $\# h_{i}$ and $\# g_{i}$ are number of elements in $h$ and $g$ respectively, then

1) if $s_{1}>s_{2}$ then $d_{1}$ is superior to $d_{2}$, denoted by $d_{1} \succ d_{2}$

2) if $s_{1}=s_{2}$, if $p_{1}>p_{2}$ then $d_{1}$ is superior to $d_{2}$, denoted by $d_{1} \succ d_{2}$ if $p_{1}=p_{2}$ then $d_{1}$ is equivalent to $d_{2}$, denoted by $d_{1} \sim d_{2}$.

\section{Z-Score Functions of Dual Hesitant Fuzzy Set}

The score function of DHFE plays an important role in determining a single value to represent the element. We propose a $\mathrm{z}$-score function consisting of the $\mathrm{z}$-arithmetic mean, z-geometric mean and $z$-harmonic mean. The proposed DHFE novel z-score function considers five key aspects:

a) Degree of hesitant for membership and non-membership

The degree of hesitant of a DHFE depends on the number of membership and non-membership functions of the element. The more numbers of element in the DHFE, the higher degree of hesitant.

b) Deviation degree for membership and non-membership

The deviation degree of membership and non-membership for DHFE is a measure of the amount of variation or distribution of a set of value. A low deviation degree indicates that its elements tend to approach the mean of the set, while a high deviation degree indicates that its elements are spread over a wider range.

c) Parameter $\alpha:$ the importance of the hesitant degree of DHFE.

This degree explains the importance of the level of hesitant for membership and non-membership functions in determining the degree of mean arithmetic score, mean geometric and mean harmonic. $\alpha=[0,1]$ is the degree used by decision makers to determine the degree of this score. The higher the degree, the more important the level of DHFE hesitant in determining the score for DHFE.

d) Parameter $\beta$ : the importance of the deviation degree of DHFE.

This degree explains the importance of deviation 
degrees for membership and non-membership functions in determining the arithmetic- $z$ mean score degree, z-geometric mean and z-harmonic mean. $\beta=[0,1]$ is the degrees used by decision makers to determine the degree of a $\mathrm{Z}$-score. The higher the degree, the more important the deviation degree in determining the $\mathrm{z}$-score for DHFE.

e) Parameter $\vartheta:$ the importance of membership or non-membership degree.

This parameter explains the importance of membership degree (positive view) and non-membership (negative view) degree in determining the arithmetic-z mean score degree, z-geometric mean and z-harmonic mean. $\vartheta=[0,1]$ is used by decision makers to determine the z-score value. If $\vartheta=0.5$, it indicates that the degree of membership is as important as the degree of non-membership in determining the score for DHFE. If $\vartheta=[0,0.5)$, it indicates that the non-membership degree (negative view) is more important in determining the z-score for DHFE as suggested by Ren, Xu, and Wang [16]. Whereas when $\vartheta=(0.5,1]$, it shows that membership degree (positive view) is more important than non-membership degree.

Definition 5. Suppose $d_{i}=\left(h_{i}, g_{i}\right)$ is a DHFE. Then the following is called the $\mathrm{z}$-arithmetic mean score

$$
\begin{aligned}
s_{A M}\left(d_{i}\right)= & (\vartheta)\left(\bar{\gamma}\left(d_{i}\right) \times\left(1-h_{\gamma}\left(d_{i}\right)\right)^{\alpha} \times\left(1-\hat{s}_{\gamma}\left(d_{i}\right)\right)^{\beta}\right)- \\
& (1-\vartheta)\left(\bar{\eta}\left(d_{i}\right) \times\left(1-h_{\eta}\left(d_{i}\right)\right)^{\alpha} \times\left(1-\hat{s}_{\eta}\left(d_{i}\right)\right)^{\beta}\right)
\end{aligned}
$$

Definition 6. Suppose $d_{i}=\left(h_{i}, g_{i}\right)$ is a HFE. Then the following is called the $\mathrm{z}$-geometric mean score

$$
\begin{array}{r}
s_{G M}=(\vartheta)\left(\breve{\gamma} \times\left(1-h_{\gamma}\left(d_{i}\right)\right)^{\alpha} \times\left(1-\hat{s}_{\gamma}\left(d_{i}\right)\right)^{\beta}\right)- \\
(1-\vartheta)\left(\breve{\eta} \times\left(1-h_{\eta}\left(d_{i}\right)\right)^{\alpha} \times\left(1-\hat{s}_{\eta}\left(d_{i}\right)\right)^{\beta}\right)
\end{array}
$$

Definition 7. Suppose $d_{i}=\left(h_{i}, g_{i}\right)$ is a DHFE. Then the following is called the $\mathrm{z}$-harmonic mean score

$$
\begin{gathered}
s_{H M}=(\vartheta)\left(\tilde{\gamma} \times\left(1-h_{\gamma}\left(d_{i}\right)\right)^{\alpha} \times\left(1-\hat{s}_{\gamma}\left(d_{i}\right)\right)^{\beta}\right)- \\
(1-\vartheta)\left(\tilde{\eta} \times\left(1-h_{\eta}\left(d_{i}\right)\right)^{\alpha} \times\left(1-\hat{s}_{\eta}\left(d_{i}\right)\right)^{\beta}\right) \\
\text { where } \bar{\gamma}=\frac{\sum_{i=1}^{\# h} \gamma_{i}}{\# h} \text { and } \bar{\eta}=\frac{\sum_{i=1}^{\# g} \eta_{i}}{\# g} \text { are the arithmetic means }
\end{gathered}
$$
for membership and non-membership respectively. Meanwhile $\breve{\gamma}=\left(\prod_{i=1}^{\# h} \gamma_{i}\right)^{\frac{1}{\# h}}$ and $\breve{\eta}=\left(\prod_{i=1}^{\# g} \eta_{i}\right)^{\frac{1}{\# g}}$ are the geometric means for membership and non-membership. where $\tilde{\gamma}=\frac{\# h}{\sum_{i=1}^{\# h} \frac{1}{\gamma_{i}}}$ and $\tilde{\eta}=\frac{\# g}{\sum_{i=1}^{\# h} \frac{1}{\eta_{i}}}$ is the mean harmonic for membership and non-membership respectively. $\hat{s}_{\gamma}\left(d_{i}\right)=\sum_{i=1}^{\# h} \sqrt{\frac{(\bar{\gamma}-\gamma)^{2}}{\# h}}$ is the deviation degree for membership and $\hat{s}_{\eta}\left(d_{i}\right)=\sum_{i=1}^{\# g} \sqrt{\frac{(\bar{\eta}-\eta)^{2}}{\# g}}$ is the deviation degree for non-membership. Next, $\left.h_{\gamma}\left(d_{i}\right)\right)=\frac{\# h-1}{\chi}$ is the degree of hesitant for membership and $h_{\eta}\left(d_{i}\right)=\frac{\# g-1}{\chi}$ is the degree of hesitant for membership. The $\chi=10$ if $\# h$ $\# h$, $g \leq 10$ study is set to, but if the \#h or \# $g$ is greater than 10 , then the degree $\chi=\max (\# h, \# g)$.

For two DHFEs, $\tilde{h}_{1}$ and $\tilde{h}_{2}$, if $S\left(\tilde{h}_{1}\right) \succ S\left(\tilde{h}_{2}\right)$ then $\tilde{h}_{1}$ is higher than $\tilde{h}_{2}$ represented by $\tilde{h}_{1} \succ \tilde{h}_{2}$ and if $S\left(\tilde{h}_{1}\right)=S\left(\tilde{h}_{2}\right)$ then $\tilde{h}_{1}$ is no difference than $\tilde{h}_{2}$ represented by $\tilde{h}_{1} \sim \tilde{h}_{2}$.

Example 1 [16]. Suppose $D=\left\{d_{1}, d_{2}, d_{3}, d_{4}, d_{5}, d_{6}\right\}$ are six DHFEs in $D$ shown as follows:

$$
\begin{gathered}
d_{1}=\{(0.2,0.3),(0.1,0.2,0.3,0.4)\} \\
d_{2}=\{(0.1,0.2,0.3,0.4),(0.2,0.3)\} \\
d_{3}=\{(0.1,0.2),(0.1,0.2)\} \\
d_{4}=\{(0.4,0.5),(0.4,0.5)\} \\
d_{5}=\{(0.3,0.4),(0.1,0.2)\} \\
d_{6}=\{(0.5,0.6),(0.3,0.4)\} .
\end{gathered}
$$

Based on Definition 7, the z-arithmetic mean score can be calculated as follows,

$$
\begin{gathered}
\left.h_{\gamma}\left(d_{1}\right)\right)=\frac{2-1}{10}=0.1, \quad g_{\eta}\left(d_{i}\right)=\frac{4-1}{10}=0.3 \\
\bar{\gamma}\left(d_{1}\right)=\frac{0.2+0.3}{2}=0.25 \quad \bar{\eta}\left(d_{1}\right)=\frac{0.1+0.2+0.3+0.4}{4} \\
\hat{s}_{\gamma}\left(d_{i}\right)=\sqrt{\frac{(0.25-0.2)^{2}+(0.3-0.2)^{2}}{2}}=0.05 \\
\sum_{i=1}^{\# g} \sqrt{\frac{(0.25-0.1)^{2}+(0.25-0.2)^{2}+(0.25-0.3)^{2}+(0.25-0.4)^{2}}{4}} \\
=0.1118 \quad(1-0.5)\left(0.25 \times(1-0.3)^{1} \times(1-0.1118)^{1}\right) \\
=0.0292
\end{gathered}
$$


Table 1. Ranking based on z-arithmetic mean with $(\alpha, \beta)=(1,1)$

\begin{tabular}{cccccccc}
\hline & $d_{1}$ & $d_{2}$ & $d_{3}$ & $d_{4}$ & $d_{5}$ & $d_{6}$ & Ranking \\
\hline 0.1 & -0.1185 & -0.1768 & -0.1026 & -0.3078 & -0.0855 & -0.2223 & $d_{5} \succ d_{3} \succ d_{1} \succ d_{2} \succ d_{6} \succ d_{4}$ \\
0.3 & -0.0447 & -0.103 & -0.0513 & -0.1539 & 0 & -0.0684 & $d_{5} \succ d_{1} \succ d_{3} \succ d_{2} \succ d_{6} \succ d_{4}$ \\
0.5 & 0.0292 & -0.0292 & 0 & 0 & 0.0855 & 0.0855 & $d_{5} \sim d_{6} \succ d_{1} \succ d_{3} \sim d_{4} \succ d_{2}$ \\
0.7 & 0.103 & 0.0447 & 0.0513 & 0.1539 & 0.171 & 0.2394 & $d_{6} \succ d_{5} \succ d_{4} \succ d_{1} \succ d_{3} \succ d_{2}$ \\
0.9 & 0.1768 & 0.1185 & 0.1026 & 0.3078 & 0.2565 & 0.3933 & $d_{6} \succ d_{4} \succ d_{5} \succ d_{1} \succ d_{2} \succ d_{3}$ \\
\hline
\end{tabular}

Table 2. Ranking based on z-arithmetic mean with $(\alpha, \beta)=(0,1)$

\begin{tabular}{cccccccc}
\hline & $d_{1}$ & $d_{2}$ & $d_{3}$ & $d_{4}$ & $d_{5}$ & $d_{6}$ & Ranking \\
\hline 0.1 & -0.1761 & -0.1915 & -0.114 & -0.342 & -0.095 & -0.247 & $d_{5} \succ d_{3} \succ d_{1} \succ d_{2} \succ d_{6} \succ d_{4}$ \\
0.3 & -0.0842 & -0.0996 & -0.057 & -0.171 & 0 & -0.076 & $d_{5} \succ d_{1} \succ d_{3} \succ d_{2} \succ d_{6} \succ d_{4}$ \\
0.5 & 0.0077 & -0.0077 & 0 & 0 & 0.095 & 0.095 & $d_{5} \sim d_{6} \succ d_{1} \succ d_{3} \sim d_{4} \succ d_{2}$ \\
0.7 & 0.0996 & 0.0842 & 0.057 & 0.171 & 0.19 & 0.266 & $d_{6} \succ d_{5} \succ d_{4} \succ d_{1} \succ d_{3} \succ d_{2}$ \\
0.9 & 0.1915 & 0.1761 & 0.114 & 0.342 & 0.285 & 0.437 & $d_{6} \succ d_{4} \succ d_{5} \succ d_{1} \succ d_{2} \succ d_{3}$ \\
\hline
\end{tabular}

Table 3. Ranking based on z-geometric mean with $(\alpha, \beta)=(1,1)$

\begin{tabular}{cccccccc}
\hline & $d_{1}$ & $d_{2}$ & $d_{3}$ & $d_{4}$ & $d_{5}$ & $d_{6}$ & Ranking \\
\hline 0.1 & -0.1029 & -0.1747 & -0.0967 & -0.3059 & -0.0792 & -0.2197 & $d_{5} \succ d_{3} \succ d_{1} \succ d_{2} \succ d_{6} \succ d_{4}$ \\
0.3 & -0.0335 & -0.1053 & -0.0484 & -0.1529 & 0.0042 & -0.0668 & $d_{5} \succ d_{1} \succ d_{3} \succ d_{2} \succ d_{6} \succ d_{4}$ \\
0.5 & 0.0359 & -0.0359 & 0 & 0 & 0.0876 & 0.0861 & $d_{5} \sim d_{6} \succ d_{1} \succ d_{3} \sim d_{4} \succ d_{2}$ \\
0.7 & 0.1053 & 0.0335 & 0.0484 & 0.1529 & 0.1711 & 0.239 & $d_{6} \succ d_{5} \succ d_{4} \succ d_{1} \succ d_{3} \succ d_{2}$ \\
0.9 & 0.1747 & 0.1029 & 0.0967 & 0.3059 & 0.2545 & 0.3919 & $d_{6} \succ d_{4} \succ d_{5} \succ d_{1} \succ d_{2} \succ d_{3}$ \\
\hline
\end{tabular}

Table 4. Ranking based on z-geometric mean with $(\alpha, \beta)=(0,1)$

\begin{tabular}{cccccccc}
\hline & $d_{1}$ & $d_{2}$ & $d_{3}$ & $d_{4}$ & $d_{5}$ & $d_{6}$ & Ranking \\
\hline 0.1 & -0.1185 & -0.1768 & -0.1026 & -0.3078 & -0.0855 & -0.2223 & $d_{5} \succ d_{3} \succ d_{1} \succ d_{2} \succ d_{6} \succ d_{4}$ \\
0.3 & -0.0447 & -0.103 & -0.0513 & -0.1539 & 0 & -0.0684 & $d_{5} \succ d_{1} \succ d_{3} \succ d_{2} \succ d_{6} \succ d_{4}$ \\
0.5 & 0.0292 & -0.0292 & 0 & 0 & 0.0855 & 0.0855 & $d_{5} \sim d_{6} \succ d_{1} \succ d_{3} \sim d_{4} \succ d_{2}$ \\
0.7 & 0.103 & 0.0447 & 0.0513 & 0.1539 & 0.171 & 0.2394 & $d_{6} \succ d_{5} \succ d_{4} \succ d_{1} \succ d_{3} \succ d_{2}$ \\
0.9 & 0.1768 & 0.1185 & 0.1026 & 0.3078 & 0.2565 & 0.3933 & $d_{6} \succ d_{4} \succ d_{5} \succ d_{1} \succ d_{2} \succ d_{3}$ \\
\hline
\end{tabular}

Table 5. Ranking based on z-harmonic mean with $(\alpha, \beta)=(1,1)$

\begin{tabular}{cccccccc}
\hline & $d_{1}$ & $d_{2}$ & $d_{3}$ & $d_{4}$ & $d_{5}$ & $d_{6}$ & Ranking \\
\hline 0.1 & -0.1185 & -0.1768 & -0.1026 & -0.3078 & -0.0855 & -0.2223 & $d_{5} \succ d_{3} \succ d_{1} \succ d_{2} \succ d_{6} \succ d_{4}$ \\
0.3 & -0.0447 & -0.103 & -0.0513 & -0.1539 & 0 & -0.0684 & $d_{5} \succ d_{1} \succ d_{3} \succ d_{6} \succ d_{2} \succ d_{4}$ \\
0.5 & 0.0292 & -0.0292 & 0 & 0 & 0.0855 & 0.0855 & $d_{5} \sim d_{6} \succ d_{1} \succ d_{3} \sim d_{4} \succ d_{2}$ \\
0.7 & 0.103 & 0.0447 & 0.0513 & 0.1539 & 0.171 & 0.2394 & $d_{6} \succ d_{5} \succ d_{4} \succ d_{1} \succ d_{3} \succ d_{2}$ \\
0.9 & 0.1768 & 0.1185 & 0.1026 & 0.3078 & 0.2565 & 0.3933 & $d_{6} \succ d_{4} \succ d_{5} \succ d_{1} \succ d_{3} \succ d_{2}$ \\
\hline
\end{tabular}

Table 6. Ranking based on z-harmonic mean with $(\alpha, \beta)=(1,0)$

\begin{tabular}{cccccccc}
\hline & $d_{1}$ & $d_{2}$ & $d_{3}$ & $d_{4}$ & $d_{5}$ & $d_{6}$ & Ranking \\
\hline 0.1 & -0.0994 & -0.181 & -0.096 & -0.32 & -0.0771 & -0.2286 & $d_{5} \succ d_{3} \succ d_{1} \succ d_{2} \succ d_{6} \succ d_{4}$ \\
0.3 & -0.0293 & -0.1109 & -0.048 & -0.16 & 0.0086 & -0.0687 & $d_{5} \succ d_{1} \succ d_{3} \succ d_{6} \succ d_{2} \succ d_{4}$ \\
0.5 & 0.0408 & -0.0408 & 0 & 0 & 0.0943 & 0.0912 & $d_{5} \sim d_{6} \succ d_{1} \succ d_{3} \sim d_{4} \succ d_{2}$ \\
0.7 & 0.1109 & 0.0293 & 0.048 & 0.16 & 0.18 & 0.2511 & $d_{6} \succ d_{5} \succ d_{4} \succ d_{1} \succ d_{3} \succ d_{2}$ \\
0.9 & 0.181 & 0.0994 & 0.096 & 0.32 & 0.2657 & 0.411 & $d_{6} \succ d_{4} \succ d_{5} \succ d_{1} \succ d_{3} \succ d_{2}$ \\
\hline
\end{tabular}


The z-score value for DHFE and the ranking order for example 1 are given according to Definition 5 - Definition 7 in Table 1-Table 6.

Table 1-Table 6 show the z-score ranking for $(\alpha, B)=(1,1)$ and $(\alpha, B)=(1,0)$ for six DHFEs respectively. When the DM thinks that both positive view (membership) and negative view (non-membership) sides are equally important $\vartheta=0.5$, the ranking order is $d_{5} \sim d_{6} \succ d_{1} \succ d_{3} \sim d_{4} \succ d_{2}$. When the DM slightly prefers the negative view $\vartheta=0.7$, the ranking order is $d_{6} \succ d_{5} \succ d_{4} \succ d_{1} \succ d_{3} \succ d_{2}$.

Following Definition 3 [15] for Example 1, the ranking order is as follows: $d_{6} \succ d_{5} \succ d_{4} \succ d_{1} \sim d_{2} \succ d_{3}$. However, this score function ignores the number of elements present for membership and non-membership DHFE. The degree of hesitant for DHFE indicates that the decision maker is hesitant in assessing an object or criterion. For example, suppose $d_{1}=\{\{0.3\},\{0.3\}\} \quad$ and $d_{2}=\{\{0.1,0.2,0.3,0.4,0.5\},\{0.2,0.3,0.4\}\} \quad$ are two DHFEs. Then the score and accuracy degree for both DHFE are the same, $s_{d_{1}}=0 \quad s_{d_{2}}=0, d_{d_{1}}=0.6$ and $d_{d_{2}}=0.6$. This explains that decision makers are hesitant to make judgments $d_{2}$ but the ranking order is the same $d_{1} \sim d_{2}$.

Based on Defintion 4[16] for Example 1, the ranking order is $d_{5} \succ d_{6} \succ d_{3} \succ d_{1} \succ d_{2} \succ d_{4}$. This suggests that extra attention need to be given to the assessments and non-membership functions are considered as negative assessments. In practice, negative judgments have more important effect than good judgments. Parameters $\theta$ may be considered as a priority step of DM rather than non-membership functions. The greater the parameter, $\theta$, the more important for DM to consider negative comments. Therefore, the parameter $\theta$ is different because DMs differ in the decision-making process. The ranking order for the z-score methods has been reserved that $d_{5}$ is the main choice for all $\vartheta=[0,0.5)$ and consistent with the first choice of ranking order based on Definition 3. Then the $\mathrm{z}$-score function is proven to be more flexible in DM decision-making. DM should decide whether to choose a positive view (membership) $\vartheta=(0.5,1]$, negative view (not membership) $\vartheta=[0,0.5)$, or both $\vartheta=[0,0.5]$ are equally important in making decisions.

\section{Z-Score Algorithm in MCDM}

In this section, we break it down into two parts, namely the problem-solving algorithm in MCDM and the numerical example to show the effectiveness of the proposed algorithm. The algorithm of z-score function in MCDM is shown as follows;

Step 1. Set up DHFS decision matrix

Step 2. Calculate the weighted z-score function

a) Weighted z-arithmetic mean

$$
S w_{A M}(D)=\sum_{i=1}^{k} w_{i} \cdot s_{A M}\left(d_{i}\right)
$$

b) Weighted z-geometric mean

$$
S w_{G M}(D)=\sum_{i=1}^{k} w_{i} \cdot s_{G M}\left(d_{i}\right)
$$

c) Weighted z-harmonic mean -

$$
S w_{H M}(D)=\sum_{i=1}^{k} w_{i} \cdot s_{H M}\left(d_{i}\right)
$$

Step 3. Rank the alternatives based on the weighted z-score function

For this section, we take numerical examples from Chen et al.[15]. Here we consider the problem of pattern recognition on the classification of building materials. Given four classes of metal materials, each represented by a hesitant dual set in the feature space $C_{1}, C_{2}, C_{3}, C_{4}$. We know there is an unknown type of metal material $\mathrm{A}$ in the set, and our goal is to explain which class belongs to A. All five possible metal materials $L=\left(l_{1}, l_{2}, l_{3}, l_{4}, l_{5}\right)$ is evaluated by the decision makers. The weighting vector for each criterion is given as follows $w_{i}=(0.4,0.22,0.18,0.2)^{T}$. The DHFS decision matrix is given as in Table 7 .

Table 7. DHFS decision matrix

\begin{tabular}{ccccc}
\hline$d_{1}$ & $C_{1}$ & $C_{2}$ & $C_{3}$ & $C_{4}$ \\
\hline$l_{1}$ & $\{(0.5,0.6),(0.3)\}$ & $\{(0.2),(0.7,0.8)\}$ & $\{(0.3,0.4),(0.5,0.6)\}$ & $\{(0.5,0.6,0.7),(0.3)\}$ \\
$l_{2}$ & $\{(0.8),(0.2)\}$ & $\{(0.6,0.7,0.8),(0.2)\}$ & $\{(0.1,0.2),(0.3)\}$ & $\{(0.2),(0.6,0.7,0.8)\}$ \\
$l_{3}$ & $\{(0.7,0.8),(0.2)\}$ & $\{(0.2,0.3,0.4),(0.5)\}$ & $\{(0.4,0.5),(0.2)\}$ & $\{(0.2,0.4),(0.6,0.6)\}$ \\
$l_{4}$ & $\{(0.3,0.4),(0.6)\}$ & $\{(0.4,0.5),(0.3,0.4)\}$ & $\{(0.3,0.4),(0.6)\}$ & $\{(0.4,0.5),(0.5)\}$ \\
$l_{5}$ & $\{(0.7),(0.3)\}$ & $\{(0.4,0.5),(0.3,0.4)\}$ & $\{(0.3),(0.5,0.6,0.7)\}$ & $\{(0.5),(0.4,0.5)\}$ \\
\hline
\end{tabular}


Table 8. Ranking based on weighted z-arithmetic mean with $(\alpha, \beta)=(1,1)$

\begin{tabular}{ccccccl}
\hline & $l_{1}$ & $l_{2}$ & $l_{3}$ & $l_{4}$ & $l_{5}$ & Ranking \\
\hline 0.1 & -0.3277 & -0.2031 & -0.2458 & -0.4289 & -0.2561 & $l_{2} \succ l_{3} \succ l_{5} \succ l_{1} \succ l_{4}$ \\
0.3 & -0.1718 & -0.0477 & -0.0972 & -0.2591 & -0.0839 & $l_{2} \succ l_{5} \succ l_{3} \succ l_{1} \succ l_{4}$ \\
0.5 & -0.0158 & 0.1077 & 0.0514 & -0.0893 & 0.0883 & $l_{2} \succ l_{5} \succ l_{3} \succ l_{1} \succ l_{4}$ \\
0.7 & 0.1402 & 0.2631 & 0.2000 & 0.0805 & 0.2604 & $l_{2} \succ l_{5} \succ l_{3} \succ l_{1} \succ l_{4}$ \\
0.9 & 0.2961 & 0.4185 & 0.3486 & 0.2503 & 0.4326 & $l_{5} \succ l_{2} \succ l_{3} \succ l_{1} \succ l_{4}$ \\
\hline
\end{tabular}

Table 9. Ranking based on weighted z-geometric mean with $(\alpha, \beta)=(1,1)$

\begin{tabular}{ccccccl}
\hline & $l_{1}$ & $l_{2}$ & $l_{3}$ & $l_{4}$ & $l_{5}$ & Ranking \\
\hline 0.1 & -0.3274 & -0.2027 & -0.2460 & -0.4286 & -0.2544 & $l_{2} \succ l_{3} \succ l_{5} \succ l_{1} \succ l_{4}$ \\
0.3 & -0.1719 & -0.0479 & -0.0986 & -0.2595 & -0.0827 & $l_{2} \succ l_{5} \succ l_{3} \succ l_{1} \succ l_{4}$ \\
0.5 & -0.0165 & 0.1070 & 0.0488 & -0.0904 & 0.0889 & $l_{2} \succ l_{5} \succ l_{3} \succ l_{1} \succ l_{4}$ \\
0.7 & 0.1389 & 0.2618 & 0.1961 & 0.0787 & 0.2606 & $l_{2} \succ l_{5} \succ l_{3} \succ l_{1} \succ l_{4}$ \\
0.9 & 0.2943 & 0.4167 & 0.3435 & 0.2478 & 0.4323 & $l_{5} \succ l_{2} \succ l_{3} \succ l_{1} \succ l_{4}$ \\
\hline
\end{tabular}

Table 10. Ranking based on weighted z-harmonic mean with $(\alpha, \beta)=(1,1)$

\begin{tabular}{ccccccc}
\hline & $l_{1}$ & $l_{2}$ & $l_{3}$ & $l_{4}$ & $l_{5}$ & Ranking \\
\hline 0.1 & -0.3270 & -0.2023 & -0.2462 & -0.4283 & -0.2528 & $l_{2} \succ l_{3} \succ l_{5} \succ l_{1} \succ l_{4}$ \\
0.3 & -0.1721 & -0.0480 & -0.1000 & -0.2599 & -0.0816 & $l_{2} \succ l_{5} \succ l_{3} \succ l_{1} \succ l_{4}$ \\
0.5 & -0.0173 & 0.1063 & 0.0462 & -0.0914 & 0.0896 & $l_{2} \succ l_{5} \succ l_{3} \succ l_{1} \succ l_{4}$ \\
0.7 & 0.1376 & 0.2606 & 0.1924 & 0.0770 & 0.2608 & $l_{5} \succ l_{2} \succ l_{3} \succ l_{1} \succ l_{4}$ \\
0.9 & 0.2924 & 0.4150 & 0.3386 & 0.2454 & 0.4320 & $l_{5} \succ l_{2} \succ l_{3} \succ l_{1} \succ l_{4}$ \\
\hline
\end{tabular}

Using the proposed algorithm, the ranking order of each alternative $L=\left(l_{1}, l_{2}, l_{3}, l_{4}, l_{5}\right)$ according to the z-arithmetic mean score, z-geometric mean and z-harmonic mean are given $(\alpha, \beta)=(1,1)$ as in Table 8 , Table 9 and Table 10 respectively.

Table 8, Table 9 and Table 10 show the same ranking order for weighted $\mathrm{z}$-arithmetic mean score, weighted z-geometric mean and weighted z-harmonic mean respectively for $(\alpha, \beta)=(1,1)$. The ranking order for $\vartheta=(0.1,0.3,0.5,0.9)$ is same for all methods in $\mathrm{z}$-score functions. However for $\vartheta=0.7$, weighted $\mathrm{z}$-arithmetic mean and weighted $\mathrm{z}$-geometric mean score rankings are $l_{2} \succ l_{5} \succ l_{3} \succ l_{1} \succ l_{4}$ while weighted $\mathrm{z}$-harmonic mean gives $l_{5} \succ l_{2} \succ l_{3} \succ l_{1} \succ l_{4}$. Meanwhile the algorithm used by Chen et al. [15] obtained $l_{2} \succ l_{5} \succ l_{3} \succ l_{1} \succ l_{4}$ which is equivalent to the algorithmic approach for the $\mathrm{z}$-score function. This shows that the proposed algorithm can solve MCDM problems.

\section{Conclusions}

In this paper, we have suggested three $\mathrm{z}$-score functions for DHFE namely z-arithmetic mean, z-geometric mean and $\mathrm{z}$-harmonic mean. These three score functions are based on five important foundations, namely:

a) Hesitant degree for membership and non-membership

b) Deviation degree for membership and non-membership: the higher the deviation degree, the less consistent the decision maker is in making an assessment of an object or criterion.

c) Parameter $\alpha$ which determines the importance of hesitant degree for DHFE.

d) Parameter $\beta$ which determines the importance of deviation degrees for membership and non-membership.

e) Parameters $\vartheta$ which determines the importance of positive views (membership) or negative views (non-membership).

We then performed a sensitive analysis to determine the z-score degree of each DHFE to verify whether the parameters have changed and it is proven that the parameters chosen play an important role in determining the ranking order for DHFE. Then a comparative analysis is performed with the existing score function and it is proven that the proposed $\mathrm{z}$-score is more flexible and able to address some of the weaknesses experienced in the existing method. We applied the proposed $\mathrm{z}$-arithmetic mean, $\mathrm{z}$-geometric mean and $\mathrm{z}$-harmonic mean to develop the proposed algorithm to solve MCDM problems, 
especially pattern recognition in the DHFS environment and it turns out that the proposed method can solve the problem given and the rankings order is consistent with the existing method.

\section{Acknowledgements}

We are very grateful to experts for their appropriate and constructive suggestions to improve this template.

\section{REFERENCES}

[1] B. Zhu, Z. Xu, and M. Xia, "Dual hesitant fuzzy sets," J. Appl. Math., vol. 2012, 2012, doi: 10.1155/2012/879629.

[2] L. . Zadeh, "Fuzzy Sets," Inf. Control, vol. 8, pp. 338-353, 1965, doi: https://doi.org/10.1016/S0019-9958(65)90241$\mathrm{X}$.

[3] K. T. Atanassov, "Intuitionistic Fuzzy Sets," Fuzzy Sets Syst., vol. 20, pp. 87-96, 1986.

[4] K. Atanassov and G. Gargov, "interval-valued intuitionistic fuzzy sets,” Fuzzy Sets Syst., vol. 31, no. 3, pp. 343-349, 1989, doi: 10.1016/S0165-0114(98)00436-9.

[5] V. Torra and Y. Narukawa, "On hesitant fuzzy sets and decision," IEEE Int. Conf. Fuzzy Syst., pp. 1378-1382, 2009, doi: 10.1109/FUZZY.2009.5276884.

[6] V. Torra, "Hesitant Fuzzy Sets," Int. J. Intell. Syst., vol. 25, pp. 529-539, 2010, doi: 10.1002/int.

[7] W. Bai, J. Ding, and C. Zhang, "Dual hesitant fuzzy graphs with applications to multi-attribute decision making," Int. J. Cogn. Comput. Eng., vol. 1, no. September, pp. 18-26, 2020, doi: 10.1016/j.ijcce.2020.09.002.

[8] S. Lalotra and S. Singh, "Knowledge measure of hesitant fuzzy set and its application in multi-attribute decision-making," Comput. Appl. Math., vol. 39, no. 2, pp. 1-31, 2020, doi: 10.1007/s40314-020-1095-y.

[9] B. Wang, J. Liang, and J. Pang, "Deviation Degree: A Perspective on Score Functions in Hesitant Fuzzy Sets," Int. J. Fuzzy Syst., vol. 21, no. 7, pp. 2299-2317, 2019, doi: 10.1007/s40815-019-00722-x.

[10] B. Li, Y. Yang, J. Su, N. Zhang, and S. Wang, "Two-sided matching model for complex product manufacturing tasks based on dual hesitant fuzzy preference information," Knowledge-Based Syst., no. xxxx, p. 104989, 2019, doi: 10.1016/j.knosys.2019.104989.

[11] G. Maity, D. Mardanya, S. K. Roy, and G. W. Weber, “A new approach for solving dual-hesitant fuzzy transportation problem with restrictions," Sadhana - Acad. Proc. Eng. Sci., vol. 44, no. 4, pp. 1-11, 2019, doi: 10.1007/s12046-018-1045-1.

[12] Z. Wu, F. Zhang, J. Sun, W. Wang, and X. Tang, "Novel parameterized utility function on dual hesitant fuzzy rough sets and its application in pattern recognition," Inf., vol. 10, no. 2, 2019, doi: 10.3390/info10020071.
[13] M. Riaz and M. R. Hashmi, "Fuzzy Parameterized Fuzzy Soft Compact Spaces with Decision-Making," Punjab Univ. J. Math., vol. 50, no. 2, pp. 131-145, 2018.

[14] Y. Xu, X. Shang, J. Wang, W. Wu, and H. Huang, "Some q-rung dual hesitant fuzzy Heronian mean operators with their application to multiple attribute group decision-making," Symmetry (Basel)., vol. 10, no. 10, 2018, doi: 10.3390/sym10100472.

[15] Y. Chen, X. Peng, G. Guan, and H. Jiang, "Approaches to multiple attribute decision making based on the correlation coefficient with dual hesitant fuzzy information," J. Intell. Fuzzy Syst., vol. 26, no. 5, pp. 2547-2556, 2014, doi: 10.3233/IFS-130926.

[16] Z. Ren, Z. Xu, and H. Wang, "Dual hesitant fuzzy VIKOR method for multi-criteria group decision making based on fuzzy measure and new comparison method," Inf. Sci. (Ny)., vol. 388-389, pp. 1-16, 2017, doi 10.1016/j.ins.2017.01.024.

[17] W. Zeng, Y. Xi, Q. Yin, and P. Guo, "Weighted dual hesitant fuzzy set and its application in group decision making," Neurocomputing, 2020, doi: 10.1016/j.neucom.2020.07.13 4.

[18] D. Liang, M. Wang, Z. Xu, and D. Liu, "Risk appetite dual hesitant fuzzy three-way decisions with TODIM," Inf. Sci. (Ny)., vol. 507, pp. 585-605, 2020, doi: 10.1016/j.ins.2018.12.017.

[19] S. K. Prabha, K. Jeyalakshmi, M. Thangaraj, and K. Subramani, "Dual-hesitant fuzzy transportation problem with ATM," J. Crit. Rev., vol. 7, no. 17, pp. 1309-1315, 2020, doi: $10.31838 /$ jcr.07.17.168.

[20] W. Li and X. Deng, "Multi-parameter Portfolio Selection Model with Some Novel Score-Deviation Under Dual Hesitant Fuzzy Environment," Int. J. Fuzzy Syst., vol. 22, no. 4, pp. 1123-1141, 2020, doi: $10.1007 / \mathrm{s} 40815-020-00835-8$.

[21] G. Jothilakshmi, S. K. Prabha, and P. Thirumurugan, "Application of heuristic method in dual-hesitant fuzzy transportation problem," Adv. Math. Sci. J., vol. 9, no. 12, pp. 11133-11140, 2020, doi: 10.37418/amsj.9.12.93.

[22] Z. Rodzi and A. G. Ahmad, "Fuzzy Parameterized Dual Hesitant Fuzzy Soft Sets and Its Application in TOPSIS," Mathematics and Statistics, vol. 8, no. 1, pp. 32-41, 2020, doi: $10.13189 / \mathrm{ms} .2020 .080104$.

[23] Z. Rodzi and A. G. Ahmad, "Application of Parameterized Hesitant Fuzzy Soft Set Theory in Decision Making," Mathematics and Statistics, vol. 8, no. 3, pp. 244-253, 2020, doi: $10.13189 / \mathrm{ms} .2020 .080302$.

[24] N. Chen, Z. Xu, and M. Xia, "The ELECTRE I multi-criteria decision-making method based on hesitant fuzzy sets," Int. J. Inf. Technol. Decis. Mak., vol. 14, no. 3, pp. 621-657, 2015, doi: 10.1142/S0219622014500187.

[25] M. Ranjbar, A. V. Kamyad, and S. Effati, "Fuzzy classification as a decision making problem in hesitant environments," Int. J. Inf. Decis. Sci., vol. 11, no. 1, pp. 22 35, 2019, doi: 10.1504/IJIDS.2019.096632.

[26] S. P. Wan, Y. L. Qin, and J. Y. Dong, "A hesitant fuzzy mathematical programming method for hybrid multi-criteria group decision making with hesitant fuzzy 
truth degrees," Knowledge-Based Syst., vol. 138, pp. 232 248, 2017, doi: 10.1016/j.knosys.2017.10.002.

[27] Y. Liu, J. C. R. Alcantud, R. M. Rodríguez, K. Qin, and L. Martínez, "Intertemporal Hesitant Fuzzy Soft Sets: Application to Group Decision Making," Int. J. Fuzzy Syst., vol. 22, no. 2, pp. 619-635, 2020, doi: 10.1007/s40815-020-00798-w.

[28] J. C. R. Alcantud and A. Giarlotta, "Necessary and possible hesitant fuzzy sets: A novel model for group decision making," Inf. Fusion, vol. 46, pp. 63-76, 2019, doi: 10.1016/j.inffus.2018.05.005.

[29] G. Deschrijver and E. E. Kerre, "On the relationship between some extensions of fuzzy set theory," Fuzzy Sets Syst., vol. 133, no. 2, pp. 227-235, 2003, doi: $10.1016 / \mathrm{S} 0165-0114(02) 00127-6$.

[30] M. Xia and Z. Xu, "Hesitant fuzzy information aggregation in decision making," Int. J. Approx. Reason., vol. 52, no. 3, pp. 395-407, 2011, doi: 10.1016/j.ijar.2010.09.002. 